\title{
Numerical solution of the Cauchy problem for the Laplace equation: A deterministic and Bayesian approach $\mathrm{X}$ International Conference on Adaptive Modeling and Simulation - ADMOS 2021
}

\author{
L. Héctor Juárez-Valencia*, Alberto Villeda ${ }^{\dagger}$, Marcos A. Capistrán ${ }^{\ddagger}$ \\ *,$\dagger$ Departamento de Matemáticas \\ Universidad Autónoma Metropolitana (UAM) \\ Campus Iztapalapa, 09340 Mexico City, Mexico \\ e-mail: hect@xanum.uam.mx - web page: http://mat.izt.uam.mx/mat/ \\ $\ddagger$ Centro de Investigación en Matemáticas A.C. (CIMAT) \\ Jalisco S/N, Col. Valenciana 36023 \\ Guanajuato, Gto., México \\ e-mail: marcos@cimat.mx - Web page: https://www.cimat.mx/
}

\begin{abstract}
The problem of determining a harmonic function in a bounded annular region from measurements on part of the boundary (Cauchy data), is called the Cauchy problem for the Laplace equation. We present a numerical study for the solution of the following problem in an annular region $\Omega$ : Given a function $V$ defined on the exterior boundary $\Gamma_{2}$, find a function $\varphi=\left.u\right|_{\Gamma_{1}}$ defined on the interior boundary $\Gamma_{1}$, which is the trace of a function $u \in H^{1}(\Omega)$ that satisfies:

$$
\begin{aligned}
-\nabla \cdot(\sigma \nabla u) & =0 \quad \text { in } \quad \Omega, \\
u & =V \quad \text { on } \quad \Gamma_{2}, \\
\frac{\partial u}{\partial \mathbf{n}} & =0 \quad \text { on } \quad \Gamma_{2} .
\end{aligned}
$$

This problem is ill-posed and arise in several applications. Many numerical solution techniques has been applied to this problem. In a previous study [1], this problem was solved in annular complex regions with a variational approach based on its reformulation as a boundary control problem, for which the cost function $\varphi$ incorporates a penalized term with the input Cauchy data. Lagrange linear finite elements are good enough to solve the forward problem.

Here, we go beyond and consider a statistical inversion computational model. We propose a model with Gaussian distributions based on Bayes formula. In particular, the a priori model is built up from Gaussian Markov random fields (GMRF) for spatial statistics [2], and we propose different precision matrices for the Cauchy problem. We take advantage of the relationship between the a priori distribution and traditional Tikhonov regularization to propose different models where smooth and non-smooth regularization is possible.

Concerning the Cauchy data $V$, we assume white noise on the measurements. So, the solution of the statistical model is given by a Gaussian posterior distribution. This distribution is explored by a MCMC sampling based on a Metropolis-Hasting algorithm known as the t-walk, [3]. We take advantage of the connection between the information of the data and the knowledge of the prior in order to make a low range analysis (see [4]) to estimate the optimal number of unknown parameters (discrete values of $\varphi$ ) with respect to the number of measurements. The numerical results obtained are excellent and consistent with the previous results obtained with the deterministic variational approach.
\end{abstract}




\section{REFERENCES}

[1] Conde-Mones, J.J. and Juárez-Valencia, L. H. and Oliveros-Oliveros, J.J. and León-Velasco. D.A. Stable numerical solution of the Cauchy problem for the Laplace equation in irregular annular regions. Numerical Methods for Partial Differential Equations, Vol. 33 (6), pp. 1799$1822,(2017)$.

[2] Bardsley, J. M. and Kaipio, J. Gassian Markov random filed priors for inverse problems. Inverse Problems \& Imaging, Vol. 7 (2), pp. 397-416, (2013).

[3] Christen, J. A. and Fox, C. A general purpose sampling algorithm for continuous distributions (the t-walk). Bayesian Analysis. Vol 5 (2), pp. 263-281, (2010).

[4] Spantini, A., Solonen, A., Cui, T., Martin, J., Tenorio, L. and Marzouk, Y. Optimal low-rank approximations of Bayesian linear inverse problems. SIAM Journal on Scientific Computing, Vol 37 (6), pp. A2451-A2487, (2015).
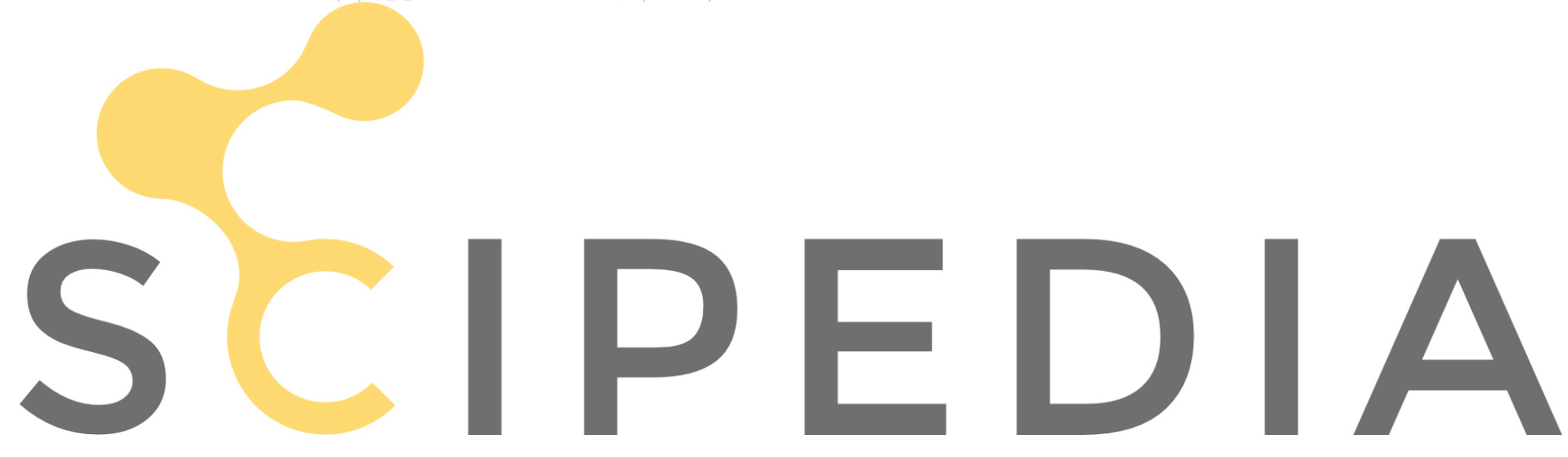